\title{
On the Dimension of the Group of Projective Transformations of Closed Randers and Riemannian Manifolds
}

Vladimir S. MATVEEV

Institute of Mathematics, Friedrich-Schiller-Universität Jena, 07737 Jena, Germany

E-mail: vladimir.matveev@uni-jena.de

URL: http://users.minet.uni-jena.de/ matveev/

Received January 18, 2012, in final form February 21, 2012; Published online February 23, 2012

http://dx.doi.org/10.3842/SIGMA.2012.007

Abstract. We construct a counterexample to Theorem 2 of [Rafie-Rad M., Rezaei B., SIGMA 7 (2011), 085, 12 pages].

Key words: Finsler metrics; Randers metrics; projective transformations

2010 Mathematics Subject Classification: 53C60; 53B40; 53A20

\section{Introduction}

A Randers metric is a Finsler metric (on a manifold $M$ ) of the form

$$
F(x, \xi)=\sqrt{g(\xi, \xi)}+\omega(\xi)
$$

where $g=g_{i j}$ is a Riemannian metric and $\omega=\omega_{i}$ is an 1-form. The assumption that $F$ given by (1) is indeed a Finsler metric is equivalent to the condition that the $g$-norm of $\omega$ is less than one. Within the whole paper we assume that all objects we consider are at least $C^{2}$-smooth.

Two Finsler metrics $F$ and $\bar{F}$ are projectively equivalent, if every forward-geodesic of $F$ is a forward-geodesic of $\bar{F}$. Within our paper we will always assume that the dimension of $M$ is at least two, since in dimension one all metrics are projectively equivalent. By a projective transformation of $(M, F)$ we understand a diffeomorphism $a: M \rightarrow M$ such that pullback of $F$ is projectively equivalent to $F$. The group of projective transformations will be denoted by $\operatorname{Proj}(M, F)$; it is a Lie group of finite dimension.

In [9, Theorem 2] it was claimed that

for closed connected $(n \geq 3)$-dimensional Randers manifold $(M, F)$ of constant flag curvature $\operatorname{dim}(\operatorname{Proj}(M, F))=n(n+2)$ or $\operatorname{dim}(\operatorname{Proj}(M, F)) \leq \frac{n(n+1)}{2}$.

The goal of the present note is to construct a counterexample to this statement: we construct a Riemannian manifold of constant sectional curvature such that its group of projective transformations is $(n-1)^{2}+1=n^{2}-2 n+2$ dimensional. Clearly, for $n>4$ we have

$$
\frac{n(n+1)}{2}<n^{2}-2 n+2=(n-1)^{2}+1<(n+1)^{2}-1=n(n+2) .
$$

Of course, Riemannian manifolds of constant sectional curvature are Randers manifolds of constant flag curvature. 


\section{Example}

Let us first recall the group of the projective transformations of the standard sphere

$$
S^{n}:=\left\{\left(x_{1}, x_{2}, \ldots, x_{n+1}\right) \in \mathbb{R}^{n+1} \mid x_{1}^{2}+x_{2}^{2}+\cdots+x_{n+1}^{2}=1\right\}
$$

with the restriction of the standard Euclidean metric. For every $A \in S L_{n+1}$, consider the diffeomorphism

$$
a: S^{n} \rightarrow S^{n}, \quad a: v \mapsto \frac{A v}{\|A v\|},
$$

where $A v$ is the product of the matrix $A$ and $v \in S^{n}$ viewed as a vector in $\mathbb{R}^{n+1}$ and $\|\cdot\|$ denotes the standard norm in $\mathbb{R}^{n+1}$.

The diffeomorphism $a$ clearly takes geodesics to geodesics. Indeed, the geodesics of $g$ are great circles (the intersections of planes that go through the origin with the sphere). Since multiplication with $A$ is a linear mapping, it takes planes to planes. Since the normalisation $w \mapsto \frac{w}{\|w\|}$ takes punctured planes to their intersections with the sphere, $a$ takes great circles to great circles. Thus, a takes geodesic to geodesic.

It is wellknown (essentially, since the time of Beltrami [2], see also [5]), that all projective transformations of the sphere can be constructed by this procedure. Clearly, two matrices $A$ and $A^{\prime} \in S L_{n+1}$ generate the same projective transformation if and only if $A^{\prime}= \pm A$ (of course, $A$ and $-A$ can simultaneosly lie in $S L_{n+1}$ only if $n$ is odd).

Let us now construct a quotient of the standard sphere modulo a (finite, freely acting) subgroup of the isometry group, such that the group of projective transformations of this quotient has dimension $n^{2}-2 n+2$.

Take $n \geq 2$ and consider the orthogonal transformation of $\mathbb{R}^{n+1}$ whose matrix $B \in O_{n+1}$ is blockdiagonal such that the first $2 \times 2$-block equals $\left(\begin{array}{cc}0 & 1 \\ -1 & 0\end{array}\right)$ and the second $(n-1) \times(n-1)$ dimensional block equals $-\mathrm{id}=\operatorname{diag}(-1, \ldots,-1)$ :

$$
B=\left(\begin{array}{cccccc}
0 & 1 & & & & \\
-1 & 0 & & & & \\
& & -1 & & & \\
& & & -1 & & \\
& & & & \ddots & \\
& & & & & -1
\end{array}\right)
$$

The restriction of this orthogonal transformation to the sphere will be denoted by $b$. Clearly, $b: S^{n} \rightarrow S^{n}$ is an involution with no fixed points preserving the metric of the sphere.

Consider now the subgroup (isomorphic to $\mathbb{Z}_{2}$ ) of the isometry group of the sphere generated by the isometry $b$, and the quotient $M:=S^{n} / \mathbb{Z}_{2}$ with the induced metric which we denote by $g$. Let us now show that the group of the projective transformations of $(M, g)$ has dimension $n^{2}-2 n+2$.

We first construct a $\left(n^{2}-2 n+2\right)$-dimensional subgroup of $\operatorname{Proj}(M, g)$. For every $A \in S L_{n+1}$ such the matrix of $A$ is blockdiagonal such that the first $2 \times 2$-block has the form $\left(\begin{array}{cc}\alpha & \beta \\ -\beta & \alpha\end{array}\right)$, where $\alpha, \beta \in \mathbb{R}$ with $\alpha^{2}+\beta^{2}>0$, and the second $(n-1) \times(n-1)$-dimensional block is an 
arbitrary matrix $\tilde{A}$ with determinant $\frac{1}{\alpha^{2}+\beta^{2}}$,

$$
A=\left(\begin{array}{ccccc}
\alpha & \beta & 0 & \ldots & 0 \\
-\beta & \alpha & 0 & \ldots & 0 \\
0 & 0 & & & \\
\vdots & \vdots & & \widetilde{A} & \\
0 & 0 & & &
\end{array}\right)
$$

For every such a matrix $A$, let us consider the projective transformations $a$ of $S^{n}$ given by (2). Since the matrices $A$ and $B$ commute, the projective transformation $a$ commutes with the isometry $b$ and induces a projective transformation of $M$. Clearly, two matrices $A$ and $A^{\prime}$ of the form (3) generate the same projective transformation of $M$ if and only if $A^{\prime} \in$

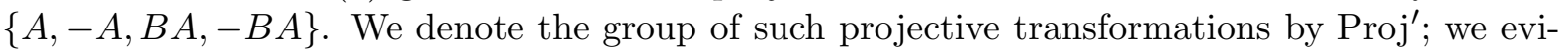
dently have

$$
\operatorname{dim}\left(\operatorname{Proj}^{\prime}\right)=\operatorname{dim}\left(G L_{n-1}\right)-1+2=(n-1)^{2}-1+2=n^{2}-2 n+2 .
$$

Let us show that the group Proj' contains (and therefore, since it is connected, coincides with) $\operatorname{Proj}_{0}(M, g)$, where $\operatorname{Proj}_{0}(M, g)$ denotes the connected component of the group of projective transformations of $(M, g)$ containing the neutral element.

Indeed, every element $\operatorname{Proj}_{0}(M, g)$ from a small neighborhood of the neutral element lies in a certain one-parametric subgroup of $\operatorname{Proj}_{0}(M, g)$. Now, one-parametric subgroups of $\operatorname{Proj}_{0}(M, g)$ are in one-to-one correspondence with projective vector fields. Since the property of a vector field to be projective is a local property, the lift of every projective vector field to $S^{n}$ is projective w.r.t. the standard metric on $S^{n}$, so it generates a 1-parameter group of projective transformations on $S^{n}$ which we denote by $a_{\tau}$. As we recalled above, for every projective transformation of $S^{n}$ there exists a matrix $A$ such that $a(x)=\frac{A x}{\|A x\|}$. We denote by $\stackrel{\tau}{A} \in S L_{n+1}$ the matrix such that $a_{\tau}(x)=\frac{\tilde{A}^{\tau} x}{\|A x\|}$.

Since the flow of such projective vector field on $S^{n}$ evidently commutes with the action of $\mathbb{Z}_{2}$ generated by $b$, for any $\tau$ the transformation $a_{\tau}$ commutes with $b$. Then, the matrices $A$ and $B$ also commute: $B \stackrel{\tau}{\mathcal{\tau}}-\stackrel{\tau}{A} B=0$. It is an easy exercise in a linear algebra to show that if a matrix $A \in S L_{n+1}$ commutes with $B$, then it has the form (3). Thus, any projective transformation of $(M, g)$ from a small neighborhood of the neutral element of $\operatorname{Proj}_{0}(M, g)$ lies in $\operatorname{Proj}^{\prime}$. Then, $\operatorname{Proj}_{0}(M, g) \subseteq \operatorname{Proj}^{\prime}$. Since Proj' is evidently connected, $\operatorname{Proj}_{0}(M, g)=\operatorname{Proj}^{\prime}$ as we claimed.

\section{The group of projective transformations of a closed Randers manifold}

Above we constructed an example of a closed manifold $(M, g)$ whose dimension of the group of projective transformations contradicts the statement of [9, Theorem 2]. The universal cover of this manifold is the standard sphere with the standard metric, and has the dimension of the group of projective transformations equal to $n(n+2)$, so the nature of our counterexample is in a certain sense topological. Of course, one can construct many other counterexamples of the same nature (i.e., such that the universal cover is the standard sphere but the group of the projective transformations has the dimension less then the dimension of the group of the projective transformations of the standard sphere but is still sufficiently big).

The following (recently proved) statement shows that such examples are essentially all possible counterexamples to [9, Theorem 2]: 
Fact (Corollary 3 of [6]). Let $(M, F)$ be a closed connected Randers manifold with $F$ given by (1). Then, at least one of the following possibilities holds:

(i) There exists a closed form $\hat{\lambda}$ such that $\operatorname{Proj}_{0}(M, F)$ consists of isometries of the Finsler metric $F(x, \xi)=\sqrt{g(\xi, \xi)}+\omega(\xi)-\hat{\lambda}(\xi)$, or

(ii) the form $\omega$ is closed and $g$ has constant positive sectional curvature.

In the case $(i)$ of Fact above, i.e., when there exists a closed form $\hat{\lambda}$ such that $\operatorname{Proj}_{0}(M, F)$ consists of isometries of the the Finsler metric $F(x, \xi)=\sqrt{g(\xi, \xi)}+\omega(\xi)-\hat{\lambda}(\xi)$, the group $\operatorname{Proj}(M, F)$ has dimension at most $\frac{n(n+1)}{2}$, see [8, Proposition 6.4]. In the case (ii) of Fact above, the group of projective transformations of $F$ coincides with the group of the projective transformations of $g$. Now, by $[4,7]$, the connected component $\operatorname{Proj}_{0}(M, g)$ of this group contains not only isometries, only if $g$ has constant positive sectional curvature. Thus, if the dimension of the group of the projective transformations of a closed $(n \geq 2)$-dimensional Randers manifold $(M, F)$ (with $F$ given by $(1))$ is greater than $\frac{n(n+1)}{2}$, then $\omega$ is a closed form and $g$ has constant positive sectional curvature. Then, the lift of $F$ to the universal cover $\tilde{M} \cong S^{n}$ of $M$ has the $n(n+2)$-dimensional group of the projective transformations.

Note that, different from [9], in the Fact above we do not require that the metric has constant flag curvature - this is an additional condition on $g$ and $\omega$ assumed in [9, Theorem 2]. Let us explain that this assumption (together with the assumption that the manifold is closed) implies that the Randers Finsler metric such that the dimension of its projective group is at least $\frac{n(n+1)}{2}$ is actually a Riemannian metric (i.e., the form $\omega$ in the formula (1) is zero).

Indeed, if the condition ( $i i)$ of Fact above holds, the Finsler metric $F$ is projectively flat (i.e., in a neighborhood of every point one can find local coordinates such that the geodesics are straight lines). If we additionally assume that the metric has constant flag curvature, the form $\omega$ must be identically zero by [1, Section 7.3$]$ so the metric is Riemannian.

Now, the condition $(i)$ of Fact above implies already that the metric $F$ is a Riemannian metric in view of $[8$, Theorem 7.1] (for $n \neq 2,4$ this fact also follows from $[3,10]$ ).

Acknowledgements. We thank O. Yakimova for useful discussions and the Deutsche Forschungsgemeinschaft (GK 1523) for partial financial support.

\section{References}

[1] Bao D., Robles C., Shen Z., Zermelo navigation on Riemannian manifolds, J. Differential Geom. 66 (2004), 377-435, math.DG/0311233.

[2] Beltrami E., Risoluzione del problema: riportare i punti di una superficie sopra un piano in modo che le linee geodetische vengano rappresentante da linee rette, Ann. di Mat. 1 (1865), no. 7, 185-204.

[3] Chen X., Deng S., A new proof of a theorem of H.C. Wang, Balkan J. Geom. Appl. 16 (2011), 25-26.

[4] Matveev V.S., Die Vermutung von Obata für Dimension 2, Arch. Math. (Basel) 82 (2004), $273-281$.

[5] Matveev V.S., Geometric explanation of the Beltrami theorem, Int. J. Geom. Methods Mod. Phys. 3 (2006), $623-629$.

[6] Matveev V.S., On projective equivalence and pointwise projective relation of Randers metrics, arXiv:1112.6143.

[7] Matveev V.S., Proof of the projective Lichnerowicz-Obata conjecture, J. Differential Geom. 75 (2007), 459-502, math.DG/0407337.

[8] Matveev V.S., Troyanov M., The Binet-Legendre metric in Finsler geometry, arXiv:1104.1647.

[9] Rafie-Rad M., Rezaei B., On the projective algebra of Randers metrics of constant flag curvature, SIGMA 7 (2011), 085, 12 pages, arXiv:1108.6127.

[10] Wang H.C., On Finsler spaces with completely integrable equations of Killing, J. London Math. Soc. 22 (1947), 5-9. 